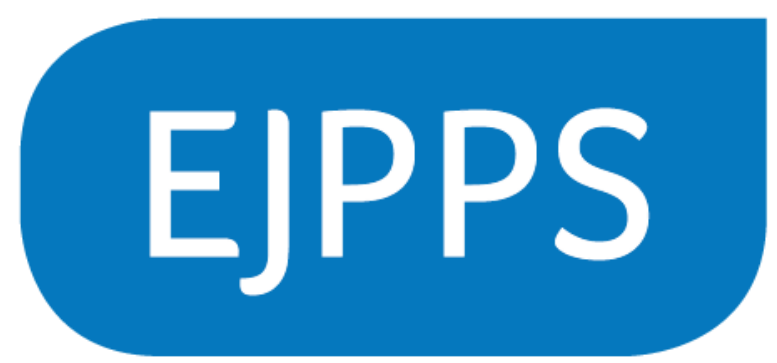

EUROPEAN JOURNAL OF
PARENTERAL AND
PHARMACEUTICAL SCIENCES

EJPPS - European Journal of Parenteral and Pharmaceutical Sciences Volume 25 Issue 1 https://www.ejpps.online/

https://doi.org/10.37521/ejpps

Investigation of the Transfer of Septum Microbial Contamination by Hypodermic Needles

T Eaton AstraZeneca, Macclesfield, UK

L Ramscar AstraZeneca, Macclesfield, UK

J Cox AstraZeneca, Macclesfield, UK

W Whyte James Watt Building South, University of Glasgow, UK

Corresponding Author: Tim Eaton, Sterile Manufacturing Specialist

AstraZeneca,

UK Operations,

Silk Road Business Park,

Macclesfield

Cheshire. SK10 2NA

England

Email: tim.eaton@astrazeneca.com

Telephone: +44(0) 1625514916 


\title{
Investigation of the Transfer of Septum Microbial Contamination by a Hypodermic Needle
}

\author{
T Eaton $^{1}$, L Ramscar ${ }^{1}$, J Cox ${ }^{1}$ and W Whyte ${ }^{2}$ \\ ${ }^{1}$ AstraZeneca, Macclesfield, UK \\ 2James Watt Building South, University of Glasgow, UK
}

\section{Summary}

The likelihood of the transfer of microbial contamination from the surface of a vial septum into the vial liquid, by penetration of a hypodermic syringe needle, has been investigated. Experimental work was carried out with vials containing sterile microbial growth media and the use of needles of three different diameters. Three different concentrations of microbes on the surface of the vial septum $(10,100$, and 1000) were used. Microbial contamination that was transferred into the growth media was determined by incubation of the vials following penetration of the septum by the needles. Contamination was detected in $87 \%$ of all the vials tested, and was generally found to increase as the concentration of septum challenge organisms and needle diameter increased.

Key words: Septum seals, hypodermic needles, multi-use vials, microbial contamination

\section{Introduction}

The risk of infection in a patient from the administration of a pharmaceutical product into the bloodstream is dependent on the product being manufactured free of microbes, and any microbes introduced during manufacture being able to survive and grow in the product. Additionally, contamination on the surface of a vial septum may be introduced into the vial when it is pierced and, furthermore, skin microbes may be transferred when a needle passes through the skin.

Information is available about the likelihood of microbial contamination during pharmaceutical product manufacturing and it is generally accepted that manufacturing carried out to the requirements of the EU GGMP ${ }^{1}$, and other applicable authority guides, will ensure minimal levels of microbial contamination. The possibility of survival and growth of microbes in products contaminated during manufacturing has also been reported ${ }^{2}$. The likelihood of the transfer of skin microbes into a patient during a subcutaneous injection, or taking blood from a vein, has been shown to occur on about $2 \%$ of occasions ${ }^{3,4}$.

The penetration of a syringe needle through a vial rubber septum may transfer micro-organisms from the surface of the septum into the vial. If conditions are favourable, the microbial contamination may multiply, and cause infections in patients. To minimise this possibility, WHO guidelines ${ }^{5}$ suggest a reduction of the concentration of septum microbes by swabbing with $70 \%$ alcohol, using gloves, and minimising the time for microbial proliferation by timely administration to the patient, and also avoiding the use of multidose vials. A key consideration associated with this risk is the likelihood of the transfer of the contamination through the septum into the vial. However, there appears to be little information available in the scientific literature about how easily this occurs. This has been investigated and reported in this article. 


\section{Experimental Methods}

\section{Preparation of vials}

The vials used in the experiments were $30 \mathrm{ml}$ capacity, with rubber septum seals secured by aluminium crimps. They were prepared with $20 \mathrm{ml}$ of tryptone soya broth (Thermo Fisher Scientific) and sterilised in an autoclave using a fluids load sterilisation cycle. Under controlled conditions, the sterile vials were sealed into three separate layers of polythene bags and gamma irradiated ( $25 \mathrm{kGy}$ ). Samples of the tryptone soya broth (TSB) were removed and tested against a range of standard test micro-organisms and their ability to support microbial growth was confirmed. Utilising sequential removal of the three layers of polythene wrappings, 180 vials were aseptically transferred into a Class II microbiological safety cabinet.

\section{Syringe Preparation}

One hundred and eighty syringes were used for the experimental work. They were divided into three groups of 60 , with each group having three different diameters of hypodermic needles $(25,18$ and 16 gauge). Shown in Table 1 is the gauge of the needles, along with their diameter, wall thickness, and surface area.

Table 1 Sizes of hypodermic needles used in experiments

\begin{tabular}{|c|c|c|c|}
$\begin{array}{c}\text { Needle } \\
\text { Gauge }\end{array}$ & $\begin{array}{c}\text { Outer Diameter } \\
(\mathrm{mm})\end{array}$ & $\begin{array}{c}\text { Wall Thickness } \\
(\mathrm{mm})\end{array}$ & $\begin{array}{c}\text { Needle Surface } \\
\text { Area } \\
\left(\mathrm{mm}^{2}\right)\end{array}$ \\
\hline $\mathbf{1 6}$ & 1.651 & 0.229 & 2.21 \\
\hline $\mathbf{1 8}$ & 1.270 & 0.216 & 1.58 \\
\hline $\mathbf{2 5}$ & 0.5144 & 0.1270 & 0.35 \\
\hline
\end{tabular}

The syringes and needles were secured in three separate layers of polythene bags and gamma irradiated (25 kGy). Sixty sterilised syringes at each needle size, (a total of 180 syringes) were then aseptically transferred into the Class II microbiological safety cabinet.

The needles with their gauge numbers and outside diameters (OD) are shown in Figure 1.

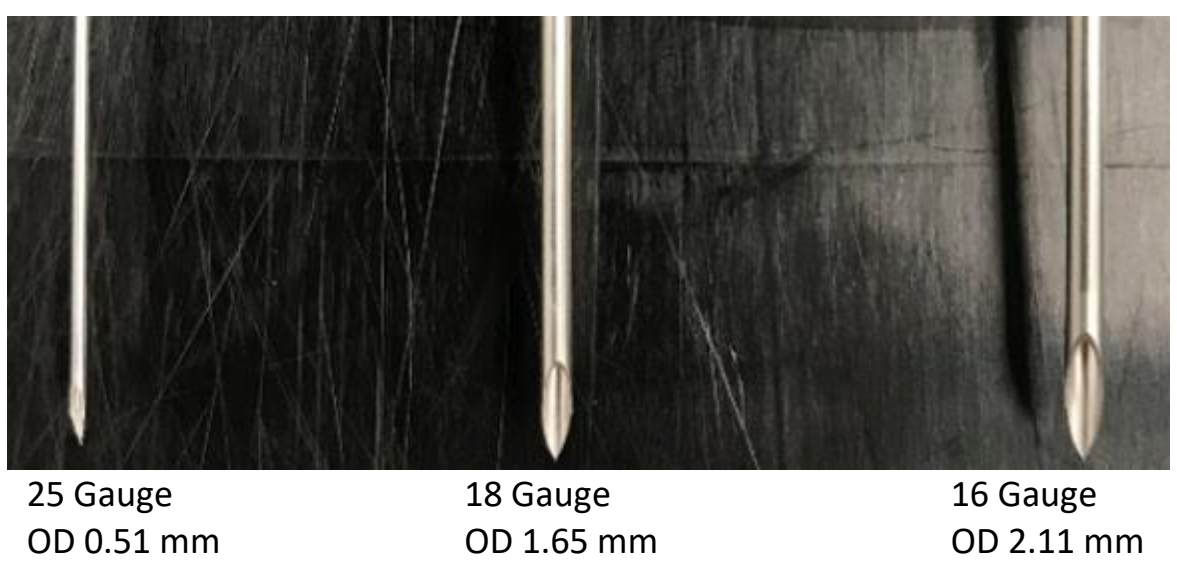

Figure 1 Syringes needles 


\section{Microbial Test Suspensions}

Microbial test suspensions were prepared at three different concentrations of 10, 100, and $1000 \mathrm{cfu}$ per $0.1 \mathrm{ml}$. These suspensions contained two types of test microbes, which were;

1.Bacillus atrophaeus (B. atrophaeus). ATCC 9372; a spore-forming organism that is able to survive adverse conditions and highly unlikely to die in the drying conditions that occurred during the experiments.

2. Micrococcus luteus (M. luteus); a common Gram-positive skin organism that was isolated from the cleanroom manufacturing facilities at AZ, Macclesfield.

\section{Test Procedure}

The needle insertion zone of the vials was the 'ribbed' area of approximately $4 \mathrm{~mm}$ diameter $\left(12.6 \mathrm{~mm}^{2}\right.$ surface area), on the outer exposed surface of the septum seals, and these were equally contaminated with $0.1 \mathrm{ml}$ of each of the six test suspensions. The six test suspensions included the two types of test organisms at each of the three concentrations. The septum seals, contaminated with the test suspension prior to drying, are shown in Figure 2. The test suspensions on the septum were then left in the Class II microbiological safety cabinet for 90 minutes to completely dry.

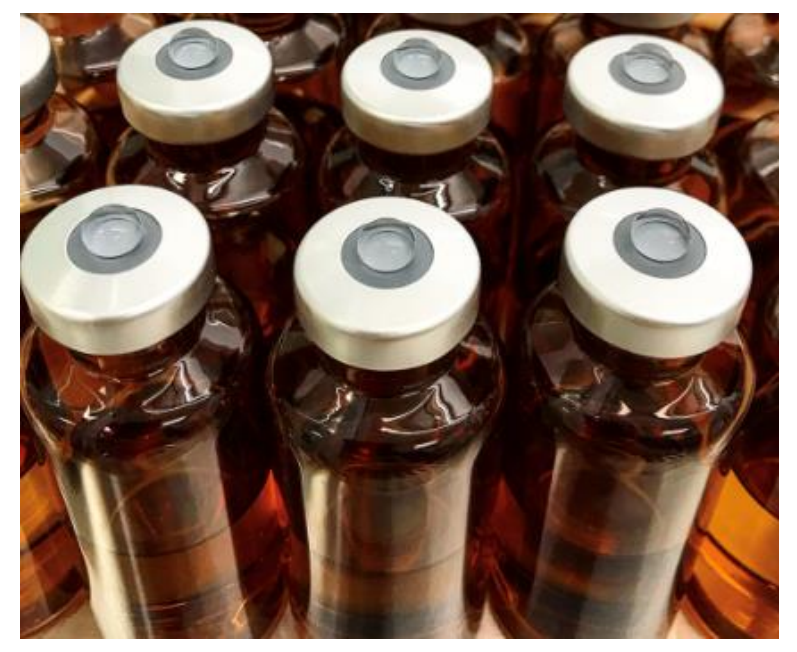

Figure 2 Vial septum seals with $0.1 \mathrm{ml}$ of microbial test suspension on the needle insertion zone

The experiments were carried out in the Class II microbiological safety cabinet. Groups of 10 vials, with three microbial concentrations of both test microbes on the vial septum, were tested with the different needle sizes. This was a total of 60 vials per needle size, and an overall total of 180 vials. The septum needle insertion zone was penetrated by a needle, and the needle was submerged in the TSB to the same depth. One rinse of the syringe and needle with the TSB was carried out, and the needle withdrawn from the septum. All vials were then incubated at $32.5^{\circ} \mathrm{C}\left( \pm 1.5^{\circ} \mathrm{C}\right)$ for five days and examined for microbial growth.

Several control experiments were carried out. The experiment carried out to ascertain the amount of vial contamination from piercing of the septum was repeated, but without any contamination of the septum seals. Also, when any growth was found in the vials, it was identified. Finally, to confirm the number test microbes placed on the septum, tryptone soya agar plates (Becton Dickinson) were inoculated with $0.1 \mathrm{ml}$ of the same suspension that was added to the septum surface and, after incubation, the number of microbial colonies counted. 


\section{Test Results}

\section{Control results}

The control experiment without contaminating the septum with test microbes resulted in no microbial contamination of the TSB. Also, the control experiment that investigated the type of growth in the vials found the growth to always be the same as the test organism used. Finally, the number of microbes placed onto the septum, in different experimental conditions, is given in Table 2. These results show that the concentrations were almost identical to the counts expected to have been added to the septum.

Table 2 Number of microbes added to the vial septums

\begin{tabular}{|c|c|c|c|c|c|c|}
\hline \multirow{3}{*}{$\begin{array}{l}\text { Needle Gauge } \\
\text { Experiment }\end{array}$} & \multicolumn{6}{|c|}{ Microbial Test Challenge } \\
\hline & \multicolumn{2}{|c|}{10 /septum } & \multicolumn{2}{|c|}{$100 /$ septum } & \multicolumn{2}{|c|}{$1000 /$ septum } \\
\hline & M. luteus & B. atrophaeus & M. luteus & B. atrophaeus & M. luteus & B. atrophaeus \\
\hline 16 & 6 & 8 & 91 & 96 & 1055 & 935 \\
\hline 18 & 11 & 13 & 89 & 122 & 990 & 1152 \\
\hline 25 & 11 & 13 & 89 & 122 & 990 & 1152 \\
\hline
\end{tabular}




\section{Growth after insertion of needle through the septum}

The results of the growth in vials caused by the two test microbes being injected through the septum and into vials are summarised in Table 3.

Table 3 Septum microbial challenges and number of contaminated vials for each needle size

\begin{tabular}{|c|c|c|c|c|c|c|c|}
\hline \multirow{3}{*}{$\begin{array}{l}\text { Needle } \\
\text { Gauge and } \\
\text { Test Details }\end{array}$} & \multicolumn{6}{|c|}{$\begin{array}{l}\text { Microbial test challenge on septum } \\
\text { Number of vials with growth (out of 10) }\end{array}$} & \multirow{3}{*}{$\begin{array}{l}\text { Average growth } \\
\text { in vial according } \\
\text { to needle gauge } \\
\text { for all } \\
\text { concentrations } \\
\text { of test organisms }\end{array}$} \\
\hline & \multicolumn{2}{|c|}{ 10/septum } & \multicolumn{2}{|c|}{$100 /$ septum } & \multicolumn{2}{|c|}{$1000 /$ septum } & \\
\hline & $\begin{array}{c}\text { M. } \\
\text { luteus }\end{array}$ & $\begin{array}{c}\text { B. } \\
\text { atrophaeus }\end{array}$ & $\begin{array}{c}\text { M. } \\
\text { luteus }\end{array}$ & $\begin{array}{c}\text { B. } \\
\text { atrophaeus }\end{array}$ & $\begin{array}{c}\text { M. } \\
\text { luteus }\end{array}$ & $\begin{array}{c}\text { B. } \\
\text { atrophaeus }\end{array}$ & \\
\hline 16 gauge & 9 & 9 & 10 & 10 & 10 & 9 & $95 \%$ \\
\hline $\begin{array}{l}\text { Average vial } \\
\text { contamination } \\
\text { per } \\
\text { concentration of } \\
\text { challenge } \\
\text { organism }\end{array}$ & \multicolumn{2}{|r|}{$90 \%$} & \multicolumn{2}{|r|}{$100 \%$} & \multicolumn{2}{|r|}{$95 \%$} & \\
\hline 18 gauge & 7 & 7 & 10 & 8 & 10 & 10 & $87 \%$ \\
\hline $\begin{array}{l}\text { Average vial } \\
\text { contamination } \\
\text { per } \\
\text { concentration of } \\
\text { challenge } \\
\text { organism }\end{array}$ & \multicolumn{2}{|r|}{$70 \%$} & \multicolumn{2}{|r|}{$90 \%$} & \multicolumn{2}{|r|}{$100 \%$} & \\
\hline 25 gauge & 8 & 5 & 9 & 9 & 10 & 7 & $80 \%$ \\
\hline $\begin{array}{l}\text { Average vial } \\
\text { contamination } \\
\text { per } \\
\text { concentration of } \\
\text { challenge } \\
\text { organism }\end{array}$ & \multicolumn{2}{|r|}{$65 \%$} & \multicolumn{2}{|r|}{$90 \%$} & \multicolumn{2}{|r|}{$85 \%$} & \\
\hline $\begin{array}{l}\text { Overall average } \\
\text { vial } \\
\text { contamination } \\
\text { per } \\
\text { concentration of } \\
\text { challenge } \\
\text { organisms for all } \\
\text { needle sizes }\end{array}$ & \multicolumn{2}{|r|}{$75 \%$} & \multicolumn{2}{|r|}{$93 \%$} & \multicolumn{2}{|r|}{$95 \%$} & - \\
\hline
\end{tabular}

It can be seen in Table 3 that 157 vials out of a total of $180(87 \%)$ were contaminated. Bacillus atrophaeus is known to survive in dry conditions and would remain viable well past the end of the test suspension drying period. This organism was included in the testing as it was unsure to what extent Micrococcus luteus, a common human skin organism, was likely to survive the drying process 
on the vial septum. However, the results indicate negligible differences in the recovery levels for both types of test organism, and so the results associated with both microbes were combined.

The results in the last row of Table 3 indicate that there is, on average, for the each of the three different sizes of needles, a trend of increased vial contamination as the concentration of challenge microbes increases. It can be seen in the bottom row that the average vial recoveries were $75 \%, 93 \%$ and $95 \%$ for the 10, 100 and 1000 microbes on the septum surface. It can also be seen in the last column of Table 1 that for the three different challenges of test microbes, there is a general decrease in the number of contaminated vials as the needle size decreases; the average vial recoveries were $95 \%, 87 \%$ and $80 \%$ for the 16,18 and 25 needle gauge sizes, respectively. It would, therefore, be reasonable to conclude that the higher the surface microbial challenge and the larger the contacting area of the needle, the greater the transfer of contamination through the septum.

\section{Discussion and Conclusions}

The transfer of microbial contamination from the surface of a vial septum into the associated vial, when the septum is penetrated by a needle, has been investigated experimentally. The septums were contaminated by different numbers of micro-organisms $(10,100$ and 1000 per septum) and pierced with three different sizes of hypodermic needle (16, 18 and 24 gauge). The needles that passed through the septum were immersed in the sterile TSB in the vial, and the microbial contamination rate of the vials determined.

For the each of the three different sizes of needles, it was found that the vial contamination rate increased as the concentration of challenge microbes on the septum surfaces increased, and the average rates were $75 \%, 93 \%$ and $95 \%$ for 10,100 , and 1000 microbes, respectively. There was also an increase in the number of contaminated vials with increasing needle size, with average contamination rates of $80 \%, 87 \%$, and $95 \%$, for the 25,18 and 16 needle gauge sizes, respectively. Overall, it is concluded that the higher the surface microbial concentrations, and the larger the diameter of the needle, the greater the risk of transfer of the contamination through the septum.

Test microbes were recovered in $65 \%$ of the vials when the lowest concentration of surface microbes (10 per septum) was present, and smallest sized needles ( 25 gauge) used. There is a reasonable likelihood that levels greater than 10 microbes will be found on vial septums after exposure to environmental contamination and handling. There is, therefore, a reasonable chance that the contents of a vial will be contaminated when its septum is pierced by a needle. These microbes will then be passed onto a patient during routine administration of pharmaceutical products.

The risk of infection to a patient from the transfer of microbes on the septum will be greater in multidose vials, as more microbes can be introduced into the vial owing to repeated piercing of the septum, and the longer time the microbes have to multiply. However, in single dose vials where the content of the vial is injected immediately, microbial growth is not possible, and this will present a much reduced risk to the patient.

Depending on the liquid formulation, micro-organisms that are deposited into the liquid in vials will either die, survive but not multiply, or multiply ${ }^{2}$. The risk to patients (including those immunosuppressed) from an occasional micro-organism is small ${ }^{6}$. Nevertheless, in formulations that provide nutrition for microbial growth, and are not sufficiently antimicrobial, microbes may multiply to a concentration as high as $10^{7} / \mathrm{ml}^{2}$. Therefore, some multi-dose vials could provide conditions which present a significant risk to the recipient. However, people can be resistant to micro-organisms introduced into their bloodstream, as occurs in normal life activities, such as when eating ${ }^{4}$ for example. However, we consider the possibility of microbial growth in a vial to be a potential risk to a patient.

In order to control the potential patient infection from contaminated vial septums, the World Health Organization (WHO) ${ }^{5}$ recommends that multidose vials are used only if there is no alternative, and discarded within 24 hours of opening, or after the time recommended by the manufacturer, if the vial does not contain antimicrobial preservatives. However, it is worth noting 
that some formulations containing preservatives will support growth of some micro-organisms of the type found in cleanrooms ${ }^{2}$.

Many vials are secured with a septum crimp that covers the full surface of the septum which ensures minimal levels of microbial contamination are maintained on the septum until it is removed prior to use. The $\mathrm{WHO}^{5}$ also recommend that, even with single dose vials, the septum is wiped with $70 \%$ alcohol (isopropyl alcohol or ethanol) on a swab or cotton-wool ball and allowing it to air dry before piercing the vial. The use of sterile gloves is also recommended. The use of $70 \%$ isopropyl alcohol to reduce surface microbial contamination on hard surfaces has been investigated ${ }^{7}$ and confirmed to be effective. However, if the level of septum contamination is high, and the method of wiping ineffective, a risk may remain.

If the likelihood of vial contamination by a needle passing through the vial's septum is considered, as well as an injection passing skin microbes into the patient, it appears that surprisingly few adverse patient reactions have been reported. This is likely to be caused by the vial liquid formulations not supporting microbial growth, and administration a short period of time after preparation. Also, people have the ability to continually and successfully deal with surprisingly large doses of microbes that invade the bloodstream ${ }^{4}$, and it is only when a substantial number of pathogens are introduced that there is a serious risk of infection. However, there is a lack of information about the degree of risk associated with these potential hazards, with scope for further research and understanding, which would be aided by the information reported in this article. 


\section{References}

1. The rules governing medicinal products in the European Union -Volume $4 \mathrm{EU}$ guidelines to good manufacturing practice - medicinal products for human and veterinary use - Annex 1 Manufacture of sterile medicinal products. European Commission, Brussels, 2008.

2. Whyte W Niven L and Bell NDS. Microbial growth in small volume pharmaceuticals. Journal of Parenteral Science and Technology 1989; 5: 208-212.

3. Whyte W, Hilditch TE and Bell NDS 1984. Microbial contamination of pharmaceutical injections at the site of administration. Journal of Clinical and Hospital Pharmacy 1984; 9: 61-67.

4. Everett, E.D. \& Hirschmann J.V. Transient bacteremia and endocarditis prophylaxis. A review. Medicine 1977; 56: 61-77.

5. World Health Organization. WHO best practices for injections and related procedures toolkit. WHO/EHT/10.02. March 2010.

6. Whyte $W$ and Eaton T. Risk from growth in products. European Journal of Parenteral and Pharmaceutical Sciences 2004; 9(1): 11-15

7. Salvage R, Hull CM, Kelly DE and Kelly SL. Use of $70 \%$ alcohol for the routine removal of microbial hard surface bioburden in life science cleanrooms. Future Microbiology 2014; 9(10):1123-30. 